\title{
Controlled placement of an individual carbon nanotube onto a microelectromechanical structure
}

\author{
P. A. Williams, S. J. Papadakis, and M. R. Falvo ${ }^{a)}$ \\ Department of Physics and Astronomy, University of North Carolina, Chapel Hill, North Carolina 27599
}

A. M. Patel

Curriculum in Applied and Materials Sciences, University of North Carolina, Chapel Hill, North Carolina 27599

M. Sinclair

Microsoft Research, Microsoft, Redmond, Washington 98052

A. Seeger

Department of Computer Science, University of North Carolina, Chapel Hill, North Carolina 27599

A. Helser

3rdTech Inc., Chapel Hill, North Carolina 27514

R. M. Taylor II

Department of Computer Science, University of North Carolina, Chapel Hill, North Carolina 27599,

Department of Physics and Astronomy, University of North Carolina, Chapel Hill,

North Carolina 27599, and Curriculum in Applied and Materials Sciences, University of North Carolina, Chapel Hill, North Carolina 27599

S. Washburn and R. Superfine

Department of Physics and Astronomy, University of North Carolina, Chapel Hill, North Carolina 27599, and Curriculum in Applied and Materials Sciences, University of North Carolina, Chapel Hill, North Carolina 27599

(Received 19 November 2001; accepted for publication 15 February 2002)

We report on the precise placement of a single carbon nanotube (CNT) onto a microlectromechanial system (MEMS) structure. Using a hybrid atomic force microscope/scanning electron microscope (AFM/SEM) system, an individual multiwalled CNT was retrieved from a cartridge by the AFM tip, translated to a MEMS device, and then placed across a gap between an actuating and a stationary structure. Progress toward a resistance versus stress/strain measurement on a CNT will be discussed, including SEM images of a MEMS structure we have designed specifically for such a measurement.

(c) 2002 American Institute of Physics. [DOI: 10.1063/1.1467701]

We are exploring the potential of bridging the micro ${ }^{1}$ and nanoscales ${ }^{2}$ in electromechanical devices through precise placement of individual carbon nanotubes $(\mathrm{CNTs})^{3}$ onto conventional microelectromechanical structures (MEMS). While MEMS consist of micron scale components and actuators, they can exhibit nanometer scale translational precision. ${ }^{4}$ Precise positioning of actuating components is therefore limited not by the translational capabilities, but by the physical size of those components. Incorporation of CNTs into MEMS allows for an order of magnitude reduction in the lateral dimension of actuating components, which will greatly enhance the MEMS performance as a probe or sensor in mechanical, ${ }^{5,6}$ electrochemical, ${ }^{7}$ biological, ${ }^{8}$ or electronic applications. ${ }^{9}$ MEMS also provide a micro laboratory for measuring physical properties of nanometer-sized objects. ${ }^{10}$

Here we report on the successful placement of an individual CNT onto a predetermined site on a MEMS structure. Using a hybrid atomic force microscope/scanning electron microscope (AFM/SEM) system, we retrieved a single CNT with the AFM tip from a CNT "cartridge," 11 transferred it to

${ }^{a)}$ Electronic mail: falvo@physics.unc.edu a desired site on a MEMS chip and then placed it across a gap between a stationary structure (reticle) and a thermal actuator (pointer). We are currently making progress toward current versus strain measurements of $\mathrm{CNTs}^{12}$ on MEMS designed for these experiments.

The experiments were performed with a combined (AFM/SEM) system (Thermomicroscopes Observer AFM mounted inside of a Hitachi S-4700 cold-cathode SEM). Note that all images presented except Fig. 4 were taken from a $45^{\circ}$ perspective relative to the sample plane. This system provides direct observation of the AFM tip movement and interaction with the sample's surface via SEM imaging, with angstrom resolution translation piezoelectric control of the AFM tip to pick up and to manipulate objects on the sample. This system is integrated with an advanced user interface for manipulation work called the nanoManipulator, ${ }^{13,14}$ allowing manual user control of the AFM tip position. The experiments described here were performed at room temperature under a vacuum of approximately $1 \times 10^{-3} \mathrm{~Pa}$.

The MEMS structure was designed using Multi User MEMS Process (MUMPs ${ }^{\circledR}$, Cronos JDS Uniphase) and made at Cronos (Research Triangle Park, NC). The MEMS components consist of several layers of polysilicon released 


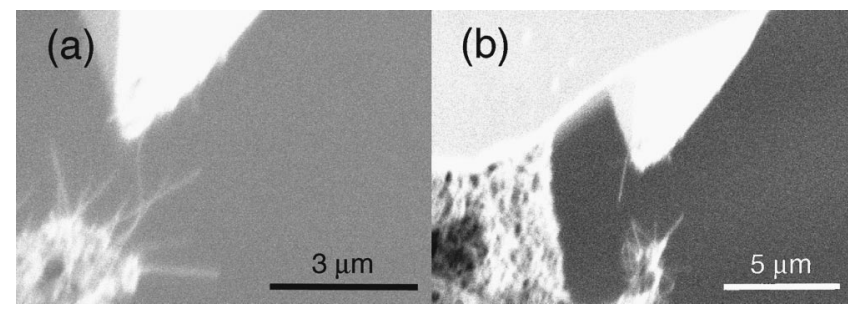

FIG. 1. (a) AFM tip (top) brought in contact with edge of copper foil CNT cartridge (lower left). (b) AFM retrieves CNT from cartridge.

from each other and the surface through a hydrofluoric acid etch of intervening oxide layers. The CNT cartridge was synthesized using coplanar copper foil electrodes. Two pieces of copper foil tape were placed on a clean glass microscope slide, separated by a $1 \mathrm{~mm}$ gap, and a $130 \mathrm{~V}-1 \mathrm{kHz}$ AC signal was applied across the gap. ${ }^{11,15}$ A suspension of CNT in isopropanol was sonicated for 30 min before applying 20 $\mu \mathrm{L}$ aliquots to the interelectrode gap area. Complete evaporation of the solvent was allowed between each aliquot. The multiwall CNTs were prepared by standard arc-discharge technique. ${ }^{16}$

The copper foil cartridge was mounted such that the CNTs were positioned perpendicular to the plane of the MEMS sample. In the SEM CNTs were observed protruding from the edge of the cartridge [Fig. 1(a)]. While observing the AFM tip in the SEM, we precisely controlled the motion of the tip to bring it down in contact with the CNTs on the cartridge [Fig. 1(a)], then slowly moved up and away from the cartridge. Upon removal of the tip from the cartridge, a single CNT had adhered to the tip, presumably through van der Waals forces [Fig. 1(b)]. The CNT was approximately 3 $\mu \mathrm{m}$ long and $50-100 \mathrm{~nm}$ in diameter.

By translating the sample, we moved the tip near the MEMS device above the $2 \mu \mathrm{m}$ gap between the pointer and the reticle [Fig. 2(a)]. The AFM tip was then lowered so that the free end of the CNT made contact with the surface of the reticle. Contact was indicated by the abrupt change of the CNT shape [Fig. 2(b)]. The CNT was then "welded" to the reticle's surface by spotting the SEM electron beam at the junction between CNT and the surface. ${ }^{10}$ The deposition of
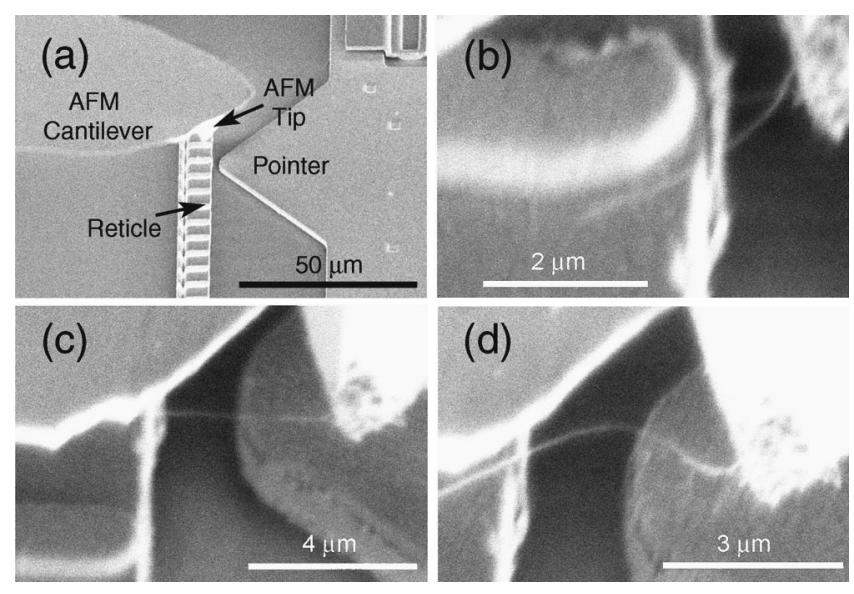

FIG. 2. (a) AFM tip with CNT brought to MEMS pointer/reticle structure. (b) CNT contacted to left side (reticle) and SEM contamination welded to the polysilicon surface. (c) AFM tip moved to the right stretching the CNT across the gap. (d) AFM tip places CNT down on pointer surface and the

CNT is pinned to this side using contamination welding. left connected to the substrate through leaf springs. A CNT
Downloaded 03 Apr 2002 to 152.2.181.189. Redistribution subject to AlP license or copyright, see http://ojps.aip.org/aplo/aplcr.jsp

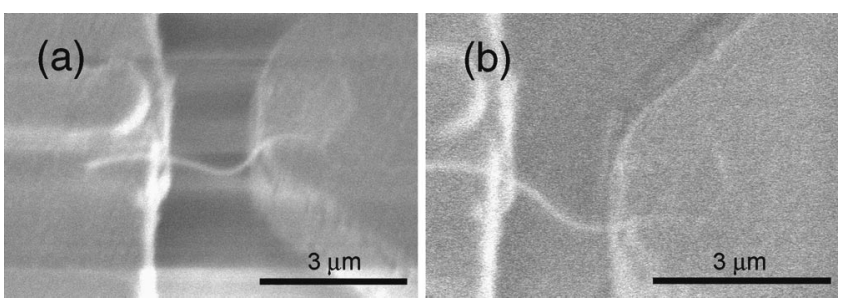

FIG. 3. (a) CNT pinned at both ends, suspended across the gap between pointer and reticle. (b) Pointer moved through AFM manipulation in order to strain CNT and test contamination welds.

carbonaceous material through SEM beam focusing (electron beam deposition or EBD) is believed to occur through dissociation of organic species in the SEM chamber. ${ }^{17,18}$ Residual organic gas molecules in the SEM chamber are ionized by the electron beam and deposited on the sample. With this method an amorphous carbon layer is built up on the junction, mechanically pinning it to the surface. After spotting the beam in this fashion for approximately $15 \mathrm{~min}$, we moved the AFM tip to the pointer such that the CNT crossed the gap between pointer and reticle. The AFM tip was then lowered onto the pointer and the junction between the CNT, and the pointer was "spot welded" for approximately 15 min. When the AFM tip was retracted from the pointer's surface, the CNT remained suspended across the $2 \mu \mathrm{m}$ gap between the pointer and reticle [Fig. 3(a)]. By applying a voltage to the pointer, we were able to show reasonable electrical contact via the carbonaceous welds from the CNT to the polysilicon surfaces. SEM images show that the pointer, $\mathrm{CNT}$, and reticle all change contrast relative to the grounded substrate due to voltage applied only to the pointer.

We tested the mechanical integrity of the SEM carbon welds by actuating the pointer relative to the reticle. To move the pointer, the AFM tip was used to push the pointer/ actuator in a direction perpendicular to the CNT. We directly observed the flexing motion of the CNT [Figs. 3(a) and 3(b)] in the SEM. The frequency of the motion was varied from about $4 \mathrm{~Hz}(1 \mu \mathrm{m}$ travel at $0.5 \mu \mathrm{m} / \mathrm{s})$ to $8 \mathrm{~Hz}(2 \mu \mathrm{m}$ travel at $0.5 \mu \mathrm{m} / \mathrm{s}$ ). Under this moderate strain, the nanotube remained securely fixed to the two sides of the gap.

To estimate the ultimate strength of the CNT weld to the MEMS device, we next used the AFM tip in an attempt to break the CNT. The AFM tip was placed in the $2 \mu \mathrm{m}$ gap adjacent to the suspended CNT and then moved into contact, applying increasing lateral force. Failure occurred in the contamination weld at one end rather than in the tube itself (tensile failure), indicating that the strength of the welds is less than that of the ultimate tensile strength of the CNT. However, it has been shown in the literature that EBD welding can be strong enough to exceed the breaking strength of CNT. ${ }^{10}$ Increasing the strength of the EBD weld requires covering more of the CNT/MEMS interface with carbonaceous material and is a matter of tuning the electron-beam parameters and the duration of deposition.

We are currently pursuing conductivity versus strain measurements using the above techniques. We have designed and fabricated MEMS structures with position and force sensitivity appropriate for such measurements (Fig. 4). The MEMS stage consists of two released stages on the right and left connected to the substrate through leaf springs. A CNT 


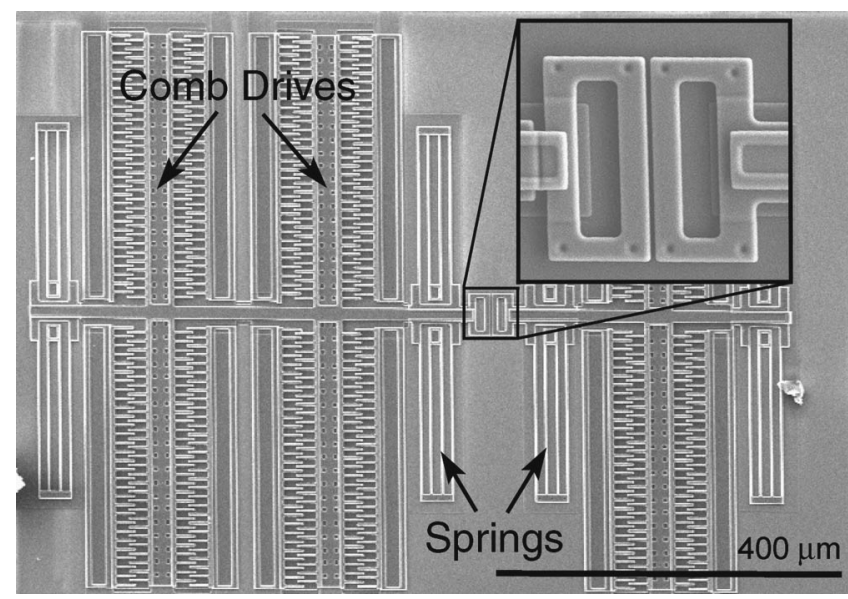

FIG. 4. Custom-designed MEMS for CNT resistance vs strain measurement. CNT will be suspended across "buckle" shown in zoomed in inset. Comb drives are used to both apply forces to the stages and measure displacements to determine strain and force (stress).

will be draped across the gap between stages (at the "buckle" near the center of Fig. 4), and pinned down with SEM carbon contamination for both mechanical rigidity and electrical contact. The left-hand stage will be pulled to the left through electrostatic actuation using one of the two sets of comb drives on the left. The other set of comb drives on the left will be used to capacitively measure the displacement of the left stage. The set of comb drives on the right will measure the displacement of the right stage. The difference between the displacements of the left and right stages will yield the strain in the suspended CNT. The displacement of the left stage multiplied by the spring constant of the leaf spring yields the tensile force on the CNT. Through independent electrical contacts to the two stages, we will simultaneously make a measurement of the CNT's resistance. The comb drives have a sensitivity of $\sim 1 \mathrm{aF} / \mathrm{nm}$. We will measure displacements with a commercial integrated circuit (Microsensors Inc., $4 \mathrm{aF} / \mathrm{Hz}^{1 / 2}$ ). This will allow us to approach nanometer resolution on strain measurements which for a 2-3 $\mu \mathrm{m}$ suspended CNT corresponds to $\sim 0.1 \%$ strain (theory and experiment show that CNT failure occurs at 5\%$10 \%$ strain $^{10}$ ). The CNT resistance will be measured using a low frequency lock-in technique.

The springs on the MEMS stages should have a low spring constant, so that they exhibit a large displacement per unit force, while still being strong enough to strain tubes to failure without breaking. Using a simple mechanical model for the stress versus strain relation ( $\sigma$ versus $\epsilon$ ) for tensile deformation of a uniform beam, we can make an estimate of the range of spring constants and ultimate tensile forces needed:

$$
\sigma=E \epsilon \quad \text { or } \quad \frac{F}{A}=E \frac{\Delta L}{L}
$$

$$
k=\frac{F}{\Delta L}=\frac{E A}{L},
$$

where $E$ is the Young's modulus of the CNT ( $1 \mathrm{TPa}$ ), $F$ is the tensile force, $A$ is its cross sectional area, $L$ is the suspended length of the CNT, and $k$ is the tensile spring constant of the CNT. For single wall CNTs and small multiwall CNTs $(r \sim 1$ $\mathrm{nm})$ of $1-2 \mu \mathrm{m}$ lengths, this corresponds to force constants on the order of $1 \mathrm{~N} / \mathrm{m}$. For a $10 \mathrm{~nm}$ diameter CNT $1 \mu \mathrm{m}$ long, $k=31 \mathrm{~N} / \mathrm{m}$. CNTs have been shown both experimentally and theoretically to be able to withstand strains up to $10 \%{ }^{10}$ This corresponds to a ultimate tensile strength of $\sim 100 \mathrm{MPa}\left(\sigma_{\mathrm{UTS}}=E \epsilon_{\mathrm{UTS}}\right)$. The force required to break a CNT will then be $F=\sigma_{\mathrm{UTS}} A$ (from Eq. 1), which corresponds to $\sim 300 \mathrm{nN}$ of force for a single wall CNT and 7500 $\mathrm{nN}$ for a $10 \mathrm{~nm}$ diameter multiwall CNT. We have chosen the spring constants on the stages of our MEMS to accommodate large maximum forces $(>10 \mathrm{mN})$ and to measure forces at strains well below $1 \%$ on the smallest CNT $(\sim 10 \mathrm{nN})$.

The Office of Naval Research, and the National Science Foundation supported this work.

${ }^{1}$ D. Bishop, P. Gammel, and C. R. Giles, Phys. Today 54, 38 (2001)

${ }^{2}$ H. G. Craighead, Science 290, 1532 (2000).

${ }^{3}$ S. Iijima, Nature (London) 354, 56 (1991).

${ }^{4}$ D. M. Freeman, MRS Bull. 26, 305 (2001)

${ }^{5}$ P. Kim and C. Lieber, Science 286, 2148 (1999).

${ }^{6}$ P. Poncharal, Z. L. Wang, D. Ugarte, and W. A. de Heer, Science 283, 1513 (1999).

${ }^{7}$ Y. Cui, Q. Q. Wei, H. K. Park, and C. M. Lieber, Science 293, 1289 (2001).

${ }^{8}$ J. Fritz, M. K. Baller, H. P. Lang, H. Rothuizen, P. Vettiger, E. Meyer, H. J. Guntherodt, C. Gerber, and J. K. Gimzewski, Science 288, 316 (2000).

${ }^{9}$ H. W. C. Postma, T. Teepen, Z. Yao, M. Grifoni, and C. Dekker, Science 293, 76 (2001).

${ }^{10}$ M. F. Yu, O. Lourie, M. J. Dyer, K. Moloni, T. F. Kelly, and R. S. Ruoff, Science 287, 637 (2000).

${ }^{11}$ H. Nishijima, S. Kamo, S. Akita, Y. Nakayama, K. I. Hohmura, S. H. Yoshimura, and K. Takeyasu, Appl. Phys. Lett. 74, 4061 (1999).

${ }^{12}$ S. Paulson, M. R. Falvo, N. Snider, A. Helser, T. Hudson, A. Seeger, R. M. Taylor, R. Superfine, and S. Washburn, Appl. Phys. Lett. 75, 2936 (1999).

${ }^{13}$ M. Guthold, M. Falvo, W. G. Matthews, S. Paulson, A. Negishi, S. Washburn, R. Superfine, F. P. Brooks, Jr., and R. M. Taylor II, J. Mol. Graphics Modell. 17, 187 (1999).

${ }^{14}$ R. M. Taylor, W. Robinett, V. L. Chi, F. P. J. Brooks, W. V. Wright, S. Williams, and E. J. Snyder, Computer Graphics: Proceedings of SIGGRAPH '93, Anaheim, CA, 1-6 August 1993, edited by J. T. Kajiya (ACM, New York, 1993), p. 127.

${ }^{15}$ X. Q. Chen, T. Saito, H. Yamada, and K. Matsushige, Appl. Phys. Lett. 78, 3714 (2001)

${ }^{16}$ T. W. Ebbesen and P. M. Ajayan, Nature (London) 358, 16 (1992).

${ }^{17}$ A. Folch, J. Servat, J. Esteve, J. Tejada, and M. Seco, J. Vac. Sci. Technol. B 14, 2609 (1996)

${ }^{18}$ H. W. P. Koops, C. Schossler, A. Kaya, and M. Weber, J. Vac. Sci. Technol. B 14, 4105 (1996). 